\title{
Kernos
}

Revue internationale et pluridisciplinaire de religion grecque antique

22 | 2009

Varia

\section{Derek Collins, Magic in the Ancient Greek World}

\section{Magali de Haro Sanchez}

Édition électronique
URL : http://journals.openedition.org/kernos/1808

DOI : 10.4000/kernos.1808

ISSN : 2034-7871

\section{Éditeur}

Centre international d'étude de la religion grecque antique

\section{Édition imprimée}

Date de publication : 1 janvier 2009

Pagination : 331-334

ISSN : 0776-3824

Référence électronique

Magali de Haro Sanchez, "Derek Collins, Magic in the Ancient Greek World », Kernos [En ligne], 22 | 2009, mis en ligne le 15 septembre 2011, consulté le 01 mai 2019. URL : http:// journals.openedition.org/kernos/1808; DOI : 10.4000/kernos.1808

Ce document a été généré automatiquement le 1 mai 2019.

Kernos 


\title{
Derek Collins, Magic in the Ancient Greek World
}

\author{
Magali de Haro Sanchez
}

\section{RÉFÉRENCE}

Derek Collins, Magic in the Ancient Greek World, Oxford, Blackwell Publishing, 2008. 1 vol. 15 国 22,5 cm, 207 p. (Blackwell Ancient Religions). ISBN : 978-1-4051-3239-8.

1 Professeur de latin et de grec à l'Université du Michigan, D. C. propose une nouvelle approche des pratiques magiques auxquelles il a par ailleurs consacré trois articles ${ }^{1}$. Deux objectifs ont guidé l'A. dans son entreprise : fournir un ouvrage de référence accessible aux non-spécialistes et, en même temps, intéresser les spécialistes dans la mesure où il ouvre de nouvelles perspectives dans l'étude de la magie grecque et de ses structures.

Intitulé Magic: What Is It and How Does It Work?, le $1^{\text {er }}$ chapitre offre une histoire des théories anthropologiques relatives à la magie telles que celles de J.G. Frazer, B. Malinowski, L. Lévy-Bruhl, E.E. Evans-Pritchard et S. Tambiah, surtout en vue de clarifier quelques concepts clés comme ceux de sympathie ou d'analogie. Dans le $2^{\mathrm{e}}$ chapitre (A Framework for Greek Magic), l'A. présente, à partir de sources littéraires, le cadre intellectuel dans lequel la magie a évolué en Grèce aux $v^{e}$ et IV siècles avant J.-C. Quoique circonscrite à l'époque classique (p. 27), son enquête commence par les épopées

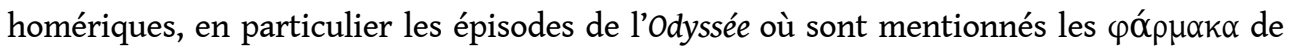
Circé et le $\mu \tilde{\omega} \lambda v$ d'Hermès. Il développe ensuite les points de vue critiques de l'auteur hippocratique du traité De la maladie sacrée et de Platon, spécialement dans les Lois, la République et le Théètète. Enfin, il insiste sur la difficulté pour les modernes de cerner la magie antique, en raison de la perception différente de la " causalité », à une époque où le monde était perçu comme un ensemble incluant tant les vivants que les invisibles (divinités, démons et morts). Une fois ces principes posés, D. C. passe en revue les

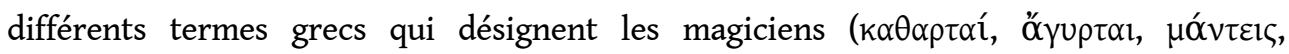




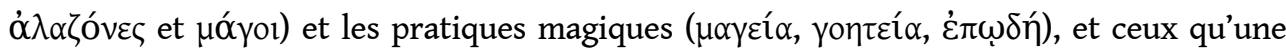
évolution sémantique rend ambigus, comme $\varphi \alpha ́$ puakov.

3 Dans le $3^{\text {e }}$ chapitre, Binding Magic and Erotic Figurines, l'A. commence par définir les défixions, leur nature, leurs supports, leurs provenances et les rituels qui les

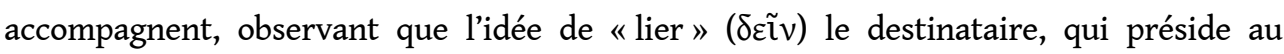
fonctionnement de la défixion, se retrouve dans les mythes, où l'action d'entraver les mouvements est souvent utilisée par les dieux ou par les hommes contre des divinités qu'ils ne peuvent tuer. Il concède toutefois que cette association d'idées ne peut être prouvée car, dans le cas des défixions, ce sont des mortels qui lient d'autres mortels. Il aborde ensuite quatre aspects de cette pratique : la demande d'assistance des morts ou des divinités infernales, l'utilisation de charaktères (symboles magiques), le démembrement des victimes et le recours aux figurines utilisées dans le rituel. D.C. prend soin de distinguer ces dernières des poupées vaudoues qui sont percées d'aiguilles ou de clous pour provoquer une douleur physique, et non pour lier à l'utilisateur de la formule chaque partie du corps de la personne objet du désir, comme dans les défixions.

4 À la lecture du titre du $4^{e}$ chapitre, Homeric Incantations, on s'attendrait à trouver des considérations sur la magie dans l'Iliade et l'Odyssée. L'A. dissipe d'emblée le malentendu en précisant qu'il étudie les vers homériques employés ultérieurement comme incantations. S'interrogeant sur les raisons du choix de ces vers dans les pratiques magiques, dans un premier temps, il se fait l'écho de la tradition rapportée par Jamblique dans la Vie de Pythagore $(25,111$ et 29,164$)$, qui invoque le principe d'analogie entre le contexte des vers homériques et la situation qui a nécessité l'emploi de la formule magique. Pourtant, D.C. constate qu'entre le $\mathrm{II}^{\mathrm{e}}$ et le $\mathrm{IV}^{\mathrm{e}}$ siècle après J.-C., la taille de la citation diminue et, avec elle, l'importance du contexte. Il montre ainsi l'importance de la métaphore dans les vers choisis pour les mots particulièrement évocateurs qu'ils

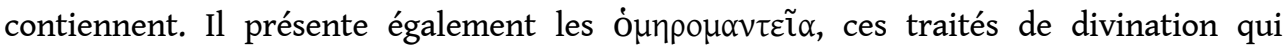
utilisent les vers d'Homère, dont on trouve un équivalent latin plus tardif: les Sortes Vergilianae. Quant au choix d'Homère, l'A. ne l'explique pas seulement par son rang de poète grec le plus illustre de l'Antiquité, mais aussi par le lien étroit noué entre la poésie et la divinité, et en particulier dans les épopées homériques, interprétation qu'on trouve notamment, écrit-il, chez Proclus.

Dans le dernier chapitre, Magic in Greek and Roman Law, il aborde enfin la difficile question de la légalité des pratiques magiques en évoquant divers cas judiciaires, ainsi que les lois ou l'absence de lois promulguées à l'encontre des personnes s'adonnant à la magie. A l'aide d'exemples éclairants, il fait remarquer qu'en Grèce et à Rome, sous la République, la condamnation vise surtout le résultat néfaste des pratiques, - assassinat ou destruction de récoltes -, alors qu'à partir des $\mathrm{III}^{\mathrm{e}}$ et $\mathrm{IV}^{\mathrm{e}}$ siècles, la simple détention d'ouvrages de magie est prohibée. Dès le $v^{e}$ siècle, l'interprétation chrétienne tentera de distinguer la magie fondée sur la nature (magie blanche), des pratiques secrètes utilisant des démons (magie noire).

6 En conclusion, l'A. reconnaît qu'on ne saurait être exhaustif dans l'étude des pratiques magiques, ajoutant que, pour un historien de la magie, il est moins important de savoir si la magie est réellement efficace que de pouvoir rendre compte des structures culturelles qui lui permettent d'exister, comme il a tenté de le faire. La fin de l'ouvrage réunit des notes fournies, un index thématique, ainsi qu'une bibliographie comprenant des monographies générales et des articles plus spécialisés. On regrettera toutefois l'absence de certaines références. Les historiens de la médecine ne manqueront pas de constater 
que J. Jouanna, dernier éditeur en date du traité hippocratique De la maladie sacrée (Paris, 2003) et spécialiste incontesté du Maître de Cos, est absent de la bibliographie. Or, J. Jouanna a notamment étudié l'attitude d'Hippocrate par rapport à la divinité en tant que cause et remède aux maladies ("Hippocrate de Cos et le sacré", Journal des savants, janvier-juin, 1989, p. 3-22; Hippocrate, Paris, 1992, p. 259-297), bien avant que ne le fasse D.C. dans son deuxième chapitre, dans lequel il utilise l'édition de H. Grensemann (Die Hippocratische Schrift: über die heilige Krankheit, Berlin, 1968), mentionnée dans les notes, mais non reprise dans la bibliographie.

On reconnaîtra à l'A. le mérite d'avoir défini des concepts clés, tels que ceux de magie sympathique, d'analogie, de magicien, de défixion, et délimité les champs sémantiques des mots grecs désignant des pratiques magiques, afin de les rendre accessibles aux nonspécialistes. On soulignera en particulier l'intérêt du $1^{\text {er }}$ chapitre qui offre un tour d'horizon rapide et complet des théories anthropologiques utiles à la compréhension des pratiques magiques et on lui saura gré de reconnaître qu'il n'a pu traiter tous les aspects de la magie. De ce fait, il n'a pas cherché à faire une synthèse, mais il a choisi des thèmes, souvent déjà abordés dans ses articles, en vue d'initier le lecteur profane aux pratiques magiques de l'Antiquité. On appréciera également le sous-chapitre consacré au traité De la maladie sacrée, dans lequel il résume les arguments de l'auteur hippocratique, tout en critiquant son attitude face aux remèdes magiques. Même s'il ne le cite pas, D.C. rejoint ici l'opinion de J. Jouanna selon laquelle l'auteur du traité ne repoussait pas totalement l'origine divine de la maladie. Cependant, dans son raisonnement, D.C. va plus loin que d'autres historiens de la magie (tel A. Bernand, Sorciers Grecs, Paris, 1991, p. 229-230), et peut-être va-t-il trop loin, en considérant que l'auteur hippocratique a tort de rejeter le recours à la magie qui aurait pu cependant offrir une réponse adéquate aux maladies supposées provoquées par les défixions (p. 38-40).

Toutefois, en dépit des efforts de son auteur, cet ouvrage n'est pas d'un abord aussi aisé qu'il pourrait l'être. Au niveau de la forme, D.C. manque de cohérence dans le découpage en sous-chapitres, faisant, par exemple, alterner dans son premier chapitre les résumés de théories anthropologiques, des définitions de concepts clés et des interprétations personnelles (Frazer and Tylor p. 3, Malinowski p. 5, Magic as Communication p. 5, Lévy-Bruhl p. 7, etc.), au risque d'égarer le lecteur. Au niveau de la typographie, sa citation de mots grecs est peu cohérente recourant tantôt, mais rarement, aux caractères grecs, tantôt à la transcription (dans l'index, par exemple, seul le verbe $\delta \varepsilon \tilde{v} v$ est écrit en grec alors que tous les autres mots sont transcrits). À la page 66, il semble même que la ligature IE du texte grec n'ait pas été correctement imprimée dans l'inscription d'une tablette de défixion attique (DTA 27, non datée, contenant $\Sigma H \Delta \operatorname{IE} \Lambda \mathrm{KI} \Sigma \Omega \Sigma$, à lire $\Sigma \omega \sigma \mathrm{k} \lambda \lambda \varepsilon 1 \delta \eta \varsigma)$, ce qui entrave la bonne compréhension de l'exemple.

9 Concernant le contenu, les spécialistes regretteront un manque de précision et parfois même de rigueur dans la présentation des sources. C'est, par exemple, le cas à la page 66, où l'A. se borne à renvoyer à J. Gager (Curse Tablets and Binding Spells from the Ancient World, Oxford, 1992) pour les tablettes de défixions et à W. Brashear («Greek Magical Papyri: an Introduction and Survey», ANRW II 18.5, 1995, p. 3380-3684) pour les papyrus, plutôt qu'aux éditions de référence des textes, lorsqu'il met en relation le principe de l'ostracisme, les défixions sur ostraca et les formules des papyrus magiques grecs à recopier sur ostraca. Une description complète des objets archéologiques cités, avec dates, provenances et lieux de conservation, et quelques illustrations auraient également aidé les lecteurs. 
10 De plus, le recul que l'A. tente de prendre par rapport à ses sources semble encore insuffisant pour les exploiter dans leur globalité, tant en ce qui concerne la forme et le contenu que le contexte culturel dans lequel elles s'inscrivent. Par exemple, dans le chapitre Homeric Incantations, D. C. présente un papyrus iatromagique (p. 109-114), le PGM XXIIa (MP3 $6001=$ BGU 4.1026, codex d'Hermopolis, IV ${ }^{e} v^{e}$ s.), qui utilise des vers d'Homère contre la fièvre, une hémorragie, des maux des seins et de la matrice, et peutêtre l'elephantiasis des anciens (affection souvent identifiée à la lèpre). Il en donne un commentaire approfondi mettant en relief l'importance des mots dans les vers utilisés comme incantations, plutôt que leur contexte dans l'épopée homérique. On remarquera

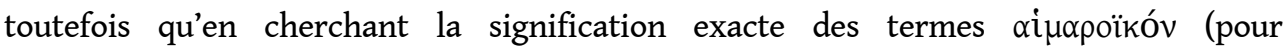

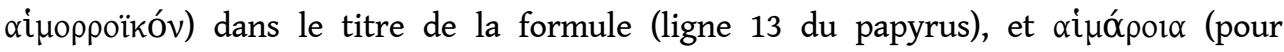

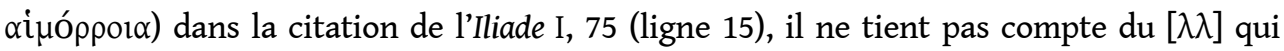

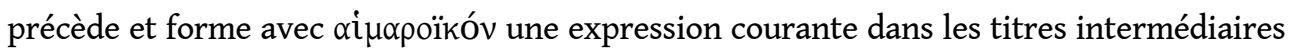
des papyrus magiques renvoyant à la formule précédente. Or, celle-ci devait être destinée à combattre une fièvre (ligne $10: \pi \rho(\grave{c} \varsigma)$ róv). S'il n'est pas impossible que l'incantation se rapporte aux hémorroïdes ou aux menstruations, comme le suggère D.C., on pourrait également l'interpréter comme le remède à une autre fièvre qualifiée d'hémorragique ou peut-être consécutive à une hémorragie, post partum par exemple. Quant au contexte culturel, le manque d'informations pose un problème, lorsqu'à la page 81 , il utilise l'anecdote mettant en scène Théophile de Chypre tirée des Miracles des Saints Cyr et Jean de Sophrone de Jérusalem ( $\mathrm{vI}^{\mathrm{e}}-\mathrm{VII}^{\mathrm{e}} \mathrm{s}$.), sans en évoquer le caractère chrétien. Selon celle-ci, une figurine de forme humaine aux pieds et aux mains percées de clous causait souffrance et paralysie aux membres correspondants de Théophile et ce n'est qu'en ôtant les clous de la figurine que la douleur de celui-ci disparut. D.C. en conclut qu'au cours du $\mathrm{VI}^{\mathrm{e}} \mathrm{s}$. après J.-C., l'effet de la défixion était compris littéralement, l'action de transpercer produisant le mal. Mais, dans un contexte chrétien, ne doit-on pas plutôt y voir une référence aux clous avec lesquels le Christ a été crucifié ? De plus, dans de telles sources, il ne faut pas négliger le jugement de valeur porté sur les pratiques magiques païennes qui devaient être présentées comme néfastes.

11 En conclusion, si D.C. a eu raison de proposer une nouvelle réflexion sur les pratiques magiques antiques, il reste encore un travail énorme de collecte, édition, réédition et exégèse à faire sur les sources épigraphiques et papyrologiques, tant profanes que chrétiennes, pour pouvoir les exploiter dans leur ensemble et les confronter avec pertinence aux sources littéraires. Complétant les Papyrus Graecae Magicae, Die griechischen Zauberpapyrus de K. Preisendanz (édition revue et complétée par A. Henrichs, Stuttgart, 1973-1974), The Greek Magical Papyrus in Translation, Including the Demotic Spells édités par H.D. Betz (2 éd. revue, Chicago, 1992), ou encore les Defixionum Tabellae d'Audollent (Paris, 1904), de nouveaux outils permettent déjà d'accéder plus aisément à la description et à la bibliographie de tels écrits, telle la base de données d'A. Kropp consacrée aux tablettes de défixions latines qui vient d'être publiée (Defixiones: ein aktuelles Corpus lateinischer Fluchtafeln, Speyer, 2008; Magische Sprachverwendung in vulgärlateinischen Fluchtafeln, avec CR-ROM, Tübingen, 2008) et le Catalogue des papyrus iatromagiques grecs, élaboré dans le cadre de la préparation de ma thèse de doctorat, disponible à la fois sous forme imprimée (dans Papyrologica Lupiensia 13, 2004, p. 39-60) et sous forme électronique sur le site du CeDoPaL (http://promethee.philo.ulg.ac.be/cedopal /Iatromagiques.htm). D'un autre côté, F. Maltomini annonce depuis plusieurs années l'élaboration d'un Corpus dei Papiri Magici Greci online (CPGM) (F. Maltomini, «P. Carlsberg. 52, p. 4 : dettagli » in C. 
Griggio, F. Vendruscolo [éd.], Suave mari magno... Studi offerti dai colleghi udinesi a Ernesto Berti, Udine, 2008, p. 183-185). En proposant un ouvrage de semi-vulgarisation, l'A. s'est heurté à la difficulté de l'exercice: faire le bilan de questions déjà bien connues des spécialistes de la magie, au risque de les lasser, et présenter des théories explicatives difficilement accessibles aux non-spécialistes car elles font appel à des notions d'anthropologie, d'histoire des religions, d'épigraphie ou de papyrologie qu'ils ne maîtrisent pas nécessairement.

\section{NOTES}

1. "Theoris of Lemnos and the Criminalization of Magic in Fourth-Century Athens ", CQ51 (2001), p. 477-493), « Nature, Cause and Agency in Greek Magic», TAPhA133 (2003), p. 17-49, et « The Magic of Homeric Verses », CPh 103 (2008), p. 211-236.

\section{AUTEURS}

\section{MAGALI DE HARO SANCHEZ}

Université de Liège 